\title{
Short Cardiac Iodine-123-Metaiodobenzylguanidine Imaging Protocol in Heart Failure
}

\author{
Takanori Arimoto, MD; Hiroyasu Sukekawa, MD; Mutsuo Harada, MD; \\ Shin Takayama, MD; Eiichiro Ikeno, MD; Kouichi Nisugi*; \\ Yasuchika Takeishi, MD**; Isao Kubota, MD**
}

\begin{abstract}
Background For assessment of cardiac sympathetic nervous activity, the conventional protocol for iodine-123metaiodobenzylguanidine (123I-MIBG) imaging requires several hours.

Methods and Results To establish whether it is possible to shorten the conventional ${ }^{123}$ I-MIBG imaging protocol, anterior planar imaging was performed in 42 heart failure (HF) patients at 5, 15 and $180 \mathrm{~min}$. The washout rate of ${ }^{123}$ I-MIBG from 5 to $15 \mathrm{~min}$ (WR5-15 min) was calculated as a novel index. WR5-15 min closely correlated with the conventional washout rate and inversely correlated with the heart to mediastinum ratio. Univariate Cox analysis revealed that rapid WR5-15 min, augmented plasma B-type natriuretic peptide level, and decreased left ventricular ejection fraction (LVEF) were predictors for cardiac events. Multivariate analysis showed WR5-15 min and LVEF were independent predictors. The cardiac event rate was markedly higher (73\%) in patients when both WR5-15 min and LVEF were abnormal.

Conclusions WR5-15 min obtained from anterior planar imaging is useful for evaluating the severity of HF and clinical outcome, and may shorten the cardiac ${ }^{123}$ I-MIBG scintigraphy protocol. (Circ J 2008; 72: 1106-1111)
\end{abstract}

Key Words: Heart failure; ${ }^{123}$ I-MIBG imaging

D espite the many life-prolonging therapies that are available for patients with heart failure (HF), the disease still has an adverse prognosis ${ }^{1-3}$ Progression of $\mathrm{HF}$ often occurs, even in the absence of overt clinical events, because HF evokes the activation of multiple neurohumoral factors, such as rennin-angiotensin-aldosterone, catecholamines, and the natriuretic peptides. Physiological findings and several noninvasive examinations, including left ventricular ejection fraction (LVEF) and B-type natriuretic peptide (BNP), for cardiac status have emerged as strong predictors of risk among patients presenting with HF and are now routinely available to clinicians ${ }^{5-16}$

We and others have characterized the prognostic value of cardiac sympathetic function assessed by iodine-123metaiodobenzylguanidine (123I-MIBG) in HF,,9,17-20 but despite the clinical utility of ${ }^{123}$ I-MIBG imaging being established, it is not used widely enough to be considered part of routine medical management, mainly because the conventional cardiac ${ }^{123}$ I-MIBG imaging protocol requires several hours, which is a contraindication in some patients.

In the present study, we examined whether it is possible to shorten the conventional ${ }^{123}$ I-MIBG imaging protocol.

(Received December 25, 2007; revised manuscript received February 15, 2008; accepted March 4, 2008)

Division of Cardiology, *Division of Radiology, Ishinomaki Red Cross Hospital, Ishinomaki and **Department of Cardiology, Pulmonology, and Nephrology, Yamagata University School of Medicine, Yamagata, Japan

Mailing address: Takanori Arimoto, MD, Cardiology Department of Internal Medicine, University of Tsukuba Graduate School of Comprehensive Human Sciences, 2-1-1 Amakubo, Tsukuba 305-8576, Japan. E-mail: takanoriarimoto@yahoo.co.jp

All rights are reserved to the Japanese Circulation Society. For permissions, please e-mail: cj@j-circ.or.jp

\section{Methods}

Study Subjects

We performed ${ }^{123}$ I-MIBG scintigraphy and measured plasma levels of BNP in 42 consecutive patients (30 men, 12 women, mean age $67 \pm 12$ years) who admitted to the Ishinomaki Red Cross Hospital for treatment of worsening chronic HF. All patients suffered from symptoms of HF, with pulmonary congestion on admission chest X-ray. Written informed consent was given by all patients, and the Institutional Review Board on Human Research approved the study protocol.

We performed conventional M-mode and 2-dimensional echocardiographic studies using standard techniques on the same day as ${ }^{123}$ I-MIBG scintigraphy, after conventional medical treatment for HF. No patients had experienced clinical symptoms or signs suggestive of acute myocardial infarction, unstable angina, or acute myocarditis in the year preceding the index admission. None had taken tricyclic antidepressants and serotonin reuptake inhibitors. Coronary angiography was performed to diagnose ischemic HF.

\section{${ }^{123}$ I-MIBG Imaging}

We performed ${ }^{123}$ I-MIBG imaging before discharge when the patients were in a stable condition. A dose of $111 \mathrm{MBq}$ of 123I-MIBG (Daiichi Radioisotope Laboratories Co, Tokyo, Japan) was administered with $10 \mathrm{ml}$ saline while resting supine after an overnight fast. All images were acquired using a $256 \times 256$ matrix and a gamma-camera with a large field of view and a low-energy, high-resolution collimator (Siemens E.CAM; Siemens Medical Systems, Inc, IL, USA). After the ${ }^{123}$ I-MIBG injection, 4-min anterior planar imaging was carried out. The planar ${ }^{123} \mathrm{I}-\mathrm{MIBG}$ images were analyzed by a region-of-interest (ROI) technique to obtain semiquantitative parameters for tracer distribution. To assess the 


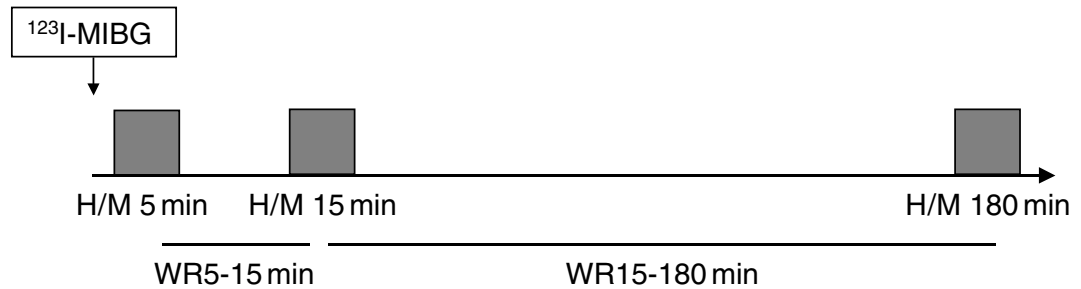

Fig 1. Protocol of iodine-123-metaiodobenzylguanidine (123I-MIBG) imaging used in the present study. We calculated the washout rate of 123 I-MIBG from 5 to $15 \mathrm{~min}$ (WR5-15 min) from the heart to mediastinum $(\mathrm{H} / \mathrm{M})$ ratio at $5 \mathrm{~min}$ and $15 \mathrm{~min}$. WR15-180 $\mathrm{min}$, washout rate of ${ }^{123} \mathrm{I}-\mathrm{MIBG}$ from 15 to $180 \mathrm{~min}$

Table 1 Clinical Background of the Patients With Heart Failure

\begin{tabular}{|c|c|c|c|c|}
\hline & $\begin{array}{c}\text { All patients } \\
\quad(n=42)\end{array}$ & $\begin{array}{c}\text { Cardiac events } \\
\quad(n=12)\end{array}$ & $\begin{array}{c}\text { No-cardiac events } \\
(n=30)\end{array}$ & $p$ value \\
\hline Age (years) & $67 \pm 12$ & $69 \pm 10$ & $67 \pm 13$ & 0.6765 \\
\hline $\operatorname{Sex}(M / F)$ & $30 / 12$ & $8 / 4$ & $22 / 8$ & 0.6657 \\
\hline NYHA functional class (II/III) & $18 / 24$ & $3 / 9$ & $15 / 15$ & 0.1391 \\
\hline Etiology & & & & 0.5838 \\
\hline Dilated cardiomyopathy & $15(36 \%)$ & $5(42 \%)$ & $10(33 \%)$ & \\
\hline Ischemic heart failure & $14(33 \%)$ & $4(33 \%)$ & $10(33 \%)$ & \\
\hline Hypertensive heart disease & $5(12 \%)$ & $1(8 \%)$ & $4(13 \%)$ & \\
\hline Valvular heart disease & $4(10 \%)$ & $2(17 \%)$ & $2(7 \%)$ & \\
\hline Other & $4(10 \%)$ & $0(0 \%)$ & $4(13 \%)$ & \\
\hline Hypertension & $15(36 \%)$ & $6(50 \%)$ & $9(30 \%)$ & 0.2217 \\
\hline Diabetes mellitus & $14(33 \%)$ & $5(42 \%)$ & $9(30 \%)$ & 0.4687 \\
\hline Hyperlipidemia & $10(24 \%)$ & $3(25 \%)$ & $7(23 \%)$ & 0.9088 \\
\hline Atrial fibrillation & $14(33 \%)$ & $4(33 \%)$ & $10(33 \%)$ & 0.9999 \\
\hline $\operatorname{LVEF}(\%)$ & $38 \pm 14$ & $32 \pm 14$ & $41 \pm 13$ & 0.0504 \\
\hline$B N P(p g / m l)$ & $444 \pm 432$ & $668 \pm 632$ & $358 \pm 288$ & 0.0332 \\
\hline \multicolumn{5}{|l|}{${ }^{123}$ I-MIBG imaging } \\
\hline$H / M 5 \min$ & $2.29 \pm 0.37$ & $2.31 \pm 0.51$ & $2.28 \pm 0.30$ & 0.7913 \\
\hline H/M $15 \mathrm{~min}$ & $2.36 \pm 0.37$ & $2.30 \pm 0.49$ & $2.38 \pm 0.32$ & 0.5280 \\
\hline H/M $180 \mathrm{~min}$ & $2.15 \pm 0.42$ & $1.99 \pm 0.41$ & $2.21 \pm 0.42$ & 0.1356 \\
\hline$W R 5-15 \min (\%)$ & $6.8 \pm 2.6$ & $8.6 \pm 1.9$ & $6.1 \pm 2.5$ & 0.0038 \\
\hline WR15-180 min (\%) & $29.0 \pm 8.8$ & $33.5 \pm 7.1$ & $27.1 \pm 8.8$ & 0.0326 \\
\hline \multicolumn{5}{|l|}{ Medications } \\
\hline ACEI and/or ARBs & $38(90 \%)$ & $12(100 \%)$ & $26(87 \%)$ & 0.1836 \\
\hline$\beta$-blockers & $27(64 \%)$ & $7(58 \%)$ & $20(67 \%)$ & 0.6106 \\
\hline Calcium-channel blockers & $15(36 \%)$ & $3(25 \%)$ & $12(40 \%)$ & 0.3594 \\
\hline Spironolactone & $12(29 \%)$ & $5(42 \%)$ & $7(23 \%)$ & 0.2348 \\
\hline Loop diuretics & $27(64 \%)$ & $10(83 \%)$ & $17(57 \%)$ & 0.1032 \\
\hline Digoxin & $18(43 \%)$ & $7(58 \%)$ & $11(37 \%)$ & 0.1999 \\
\hline
\end{tabular}

NYHA, New York Heart Association; LVEF, left ventricular ejection fraction; BNP, B-type natriuretic peptide; ${ }^{123}$ I-MIBG, iodine123-metaiodobenzylguanidine; H/M, heart to mediastinum; WR, washout rate; ACEI, angiotensin-converting enzyme inhibitor; $A R B$, angiotensin-II receptor blocker.

global myocardial kinetics of ${ }^{123} \mathrm{I}-\mathrm{MIBG}$, the ROI was drawn manually over the whole heart and a rectangular ROI over the upper mediastinum was used as a reference background region. The ${ }^{123} \mathrm{I}-\mathrm{MIBG}$ count densities of the heart $(\mathrm{H})$ and the mediastinum $(\mathrm{M})$ were calculated from the 5-, 15- and 180-min images. The heart to mediastinum (H/M) ratio of ${ }^{123} \mathrm{I}-\mathrm{MIBG}$ uptake at $5 \mathrm{~min}(\mathrm{H} / \mathrm{M} 5 \mathrm{~min}), 15 \mathrm{~min}$ (H/M $15 \mathrm{~min})$ and $180 \mathrm{~min}(\mathrm{H} / \mathrm{M} 180 \mathrm{~min})$ were calculated as previously reported $17,18,21,22$ The washout rate of 123IMIBG from 5 to $15 \mathrm{~min}$ and 15 to $180 \mathrm{~min}$ (WR5-15 min and WR15-180 $\mathrm{min}$ ) from the myocardium was calculated as $(\mathrm{H}$ at $5 \mathrm{~min}-\mathrm{H}$ at $15 \mathrm{~min}) \times 100 / \mathrm{H}$ at $5 \mathrm{~min}(\%)$ and $(\mathrm{H}$ at $15 \mathrm{~min}-\mathrm{H}$ at $180 \mathrm{~min}) \times 100 / \mathrm{H}$ at $15 \mathrm{~min}(\%)$, respectively (Fig 1). The analyses of WR5-15 min were repeated in 12 patients by the same observer and by a second observer on a separate day to determine intra- and interobserver variability, respectively. The calculated WR5-15 min was reliable, because excellent correlations were obtained from the repeated analyses $(\mathrm{R}=0.97, \mathrm{p}<0.001$ by the same observer, $\mathrm{R}=0.95, \mathrm{p}<0.001$ by the second observer).
BNP Assay

A sample of venous blood was obtained from the study subjects on the day of ${ }^{123}$ I-MIBG scintigraphy. The plasma level of BNP was measured by an immunoradiometric assay using a commercially available kit (Shionoria ${ }^{\mathrm{TM}}$ BNP, Shionogi Co, Osaka, Japan).

\section{Endpoints and Follow-up}

Patients were prospectively followed for a median period of 382 days. The end points were (1) cardiac death, defined as death from progressive HF or sudden cardiac death, and (2) progressive HF requiring hospitalization. All patients were monitored for at least 6 months.

\section{Statistical Analysis}

All values are expressed as mean \pm SD. Significance between 2 groups (with or without cardiac events) and New York Heart Association (NYHA) class II vs class III was determined by unpaired Student's t-test for continuous variables and by chi-square test for discrete variables. Values of $\mathrm{p}<0.05$ were considered significant. Univariate and multivariate analyses with the Cox proportional hazard regression 
Table 2 Results of Univariate Analysis for Predicting Cardiac Events

\begin{tabular}{|c|c|c|c|}
\hline & $H R$ & $95 \%$ CI of $H R$ & $p$ value \\
\hline Age, per 1-year increase & 1.009 & $0.961-1.058$ & $N S$ \\
\hline Male & 0.731 & $0.220-2.429$ & $N S$ \\
\hline $\begin{array}{l}\text { NYHA functional class, II vs III } \\
\text { Presence of }\end{array}$ & 0.366 & $0.099-1.358$ & $N S$ \\
\hline Hypertension & 2.095 & $0.669-6.555$ & $N S$ \\
\hline Diabetes mellitus & 1.515 & $0.478-4.799$ & $N S$ \\
\hline Hyperlipidemia & 0.952 & $0.257-3.526$ & $N S$ \\
\hline$L V E F$, per $1 \%$ increase & 0.947 & $0.899-0.999$ & 0.0445 \\
\hline $\begin{array}{l}\text { BNP, per } 1 \mathrm{pg} / \mathrm{ml} \text { increase } \\
{ }^{123} \mathrm{I}-\mathrm{MIBG} \text { imaging }\end{array}$ & 1.002 & $1.000-1.003$ & 0.0061 \\
\hline H/M $5 \mathrm{~min}^{\#}$ & 0.767 & $0.246-2.391$ & $N S$ \\
\hline H/M $15 \mathrm{~min}^{\#}$ & 1.333 & $0.422-4.208$ & $N S$ \\
\hline $\mathrm{H} / \mathrm{M} 180 \mathrm{~min}^{\#}$ & 1.864 & $0.561-6.196$ & $N S$ \\
\hline WR5-15 min * & 15.237 & $1.961-118.412$ & 0.0092 \\
\hline WR15-180 min * & 2.098 & $0.631-6.974$ & $N S$ \\
\hline
\end{tabular}

\#Inflamedian level vs supramedian level, *supramedian level vs inflamedian level.

CI, confidence interval; HR, hazard ratio; NS, no significance. Other abbreviations see in Table 1.

model were used to determine significant predictors of cardiac events. The receiver-operating characteristic (ROC) curves were constructed to illustrate various cut-off values to determine optimal sensitivity and specificity. Kaplan-Meier survival curves determined the time-dependent cumulative cardiac-event-free rates in patients stratified into 2 or 3 groups based on the values for WR5-15 min, BNP, and LVEF were analyzed by a log rank test.

\section{Results}

\section{Clinical Characteristics of Study Subjects and Patients} With Cardiac Events

Clinical characteristics, including ${ }^{123} \mathrm{I}-\mathrm{MIBG}$ scintigraphic and biochemical findings, of the 42 patients enrolled in the study are shown in Table 1: 18 patients had NYHA functional class II and 24 had class III. The etiologies of HF were identified as idiopathic dilated cardiomyopathy in 15 (36\%) patients, ischemic HF in $14(33 \%)$, hypertensive heart disease in $5(12 \%)$, valvular heart disease in $4(10 \%)$ and others in the remaining $4(10 \%)$. Hypertension, diabetes mellitus, and hyperlipidemia were identified in $15(36 \%)$, $14(33 \%)$, and $10(24 \%)$ patients, respectively. H/M 5 min, $\mathrm{H} / \mathrm{M} 15 \mathrm{~min}$ and H/M $180 \mathrm{~min}$ were $2.29 \pm 0.37$ (range 1.183.14 ), 2.36 \pm 0.37 (range 1.18-2.94) and 2.15 \pm 0.42 (range $0.98-3.30$ ), respectively. WR5-15 min and WR15-180 min were $6.8 \pm 2.6 \%$ (range $2.2-15.1 \%$ ) and $29.0 \pm 8.8 \%$ (range $14.3-49.5 \%$ ) in patients with HF. Mean plasma level of BNP was $444 \pm 432 \mathrm{pg} / \mathrm{ml}$ (range $40-1,990 \mathrm{pg} / \mathrm{ml}$ ) in patients with HF.

The patients were followed up for a median period of 382 days with regard to cardiac events. During follow-up, 9 patients were rehospitalized because of worsening congestive $\mathrm{HF} ; 2$ patients died because of progression of HF and 1 patient died of sudden cardiac death. Only 1 patient died of non-cardiac disease (renal bleeding). The comparison of the clinical characteristics of patients with or without cardiac events is shown in Table 1. Patients with cardiac events had lower LVEF and higher levels of BNP than those without cardiac events. The WR5-15 min and WR15-180 min were faster in patients with cardiac events than in those without cardiac events.

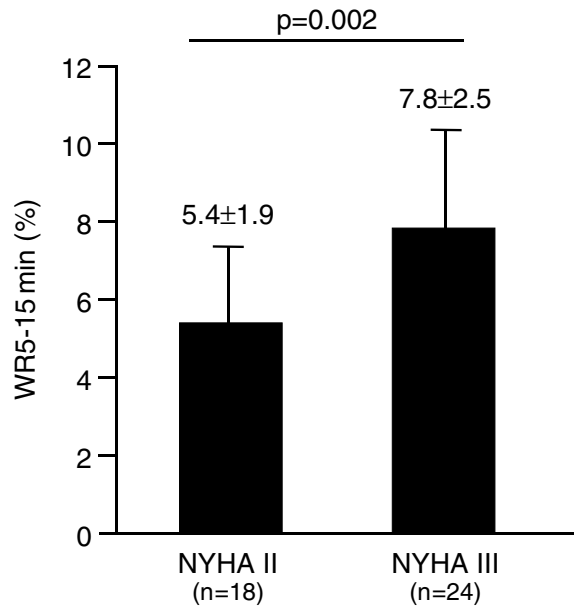

Fig 2. Washout rate of iodine-123-metaiodobenzylguanidine (123IMIBG) from 5 to $15 \mathrm{~min}$ (WR5-15 min) in the study population. NYHA, New York Heart Association.

\section{Relationships Between 123I-MIBG Imaging Parameters and NYHA Functional Class}

H/M 15 min did not differ between NYHA classes II and III $(2.41 \pm 0.27$ vs $2.32 \pm 0.43, \mathrm{p}=0.42) . \mathrm{H} / \mathrm{M} 180 \mathrm{~min}$ was lower in patients with NYHA class III than in those in class II $(2.02 \pm 0.39$ vs $2.31 \pm 0.43, \mathrm{p}=0.0263)$. WR $15-180 \mathrm{~min}$ of ${ }^{123} \mathrm{I}-\mathrm{MIBG}$ was faster in patients with NYHA class III compared with class II ( $31 \pm 9$ vs $26 \pm 8 \%, \mathrm{p}=0.0347)$. Of the novel parameters, H/M $5 \mathrm{~min}$ did not differ between NYHA classes II and III $(2.23 \pm 0.26$ vs $2.29 \pm 0.44, \mathrm{p}=0.9798)$. WR5-15 min was faster in patients with NYHA class III compared with class II $(7.8 \pm 2.5$ vs $5.4 \pm 1.9 \%, \mathrm{p}=0.002)$ as shown Fig 2.

\section{Correlations Between Our New Index and Conventional Parameters}

We examined the correlations between WR5-15 min (our novel index) and conventional scintigraphic parameters such as H/M ratio and WR15-180 min (Fig 3). WR5-15 min inversely correlated with $\mathrm{H} / \mathrm{M} 15 \mathrm{~min}(\mathrm{R}=-0.49, \mathrm{p}=0.0012)$, $\mathrm{H} / \mathrm{M} 180 \mathrm{~min}(\mathrm{R}=-0.72, \mathrm{p}<0.001)$, and was positively well correlated with WR15-180 $\min (\mathrm{R}=0.76, \mathrm{p}<0.001)$. WR5$15 \mathrm{~min}$ also positively correlated with the plasma level of $\operatorname{BNP}(\mathrm{R}=0.42, \mathrm{p}<0.01)$.

\section{Prognostic Value of ${ }^{123}$ I-MIBG Imaging, BNP and LVEF}

Univariate Cox analysis showed that WR5-15 min, BNP, and LVEF were significantly related to subsequent cardiac events (Table 2). The cut-off values for WR5-15 min, BNP, and LVEF were determined from the largest sum of sensitivity plus specificity on each ROC curve. The optimal cutoff values were $6.4 \%$ for WR5-15 min, $260 \mathrm{pg} / \mathrm{ml}$ for BNP, and $30 \%$ for LVEF. The cardiac event rate was higher in patients with a fast WR5-15 min $(\geq 6.4 \%)$ than in those with a relatively slow value $(<6.4 \%)$ (Fig $4 \mathrm{~A})$. Cardiac events occurred more often in patients with elevated BNP level $(\geq 260 \mathrm{pg} / \mathrm{ml})$ than in those with low levels $(<260 \mathrm{pg} / \mathrm{ml})$ (Fig 4B). Patients with severe reductions in left ventricular (LV) systolic function had high cardiac event rate as shown in Fig 4C. Multivariate analysis showed that only WR515 min (hazard ratio (HR) 9.789; 95\% confidence interval (CI) 1.145-83.704; $\mathrm{p}=0.0372$ ) and LVEF (HR 5.401; 95\% CI 1.397-20.883; $\mathrm{p}=0.0145$ ) remained as independent 

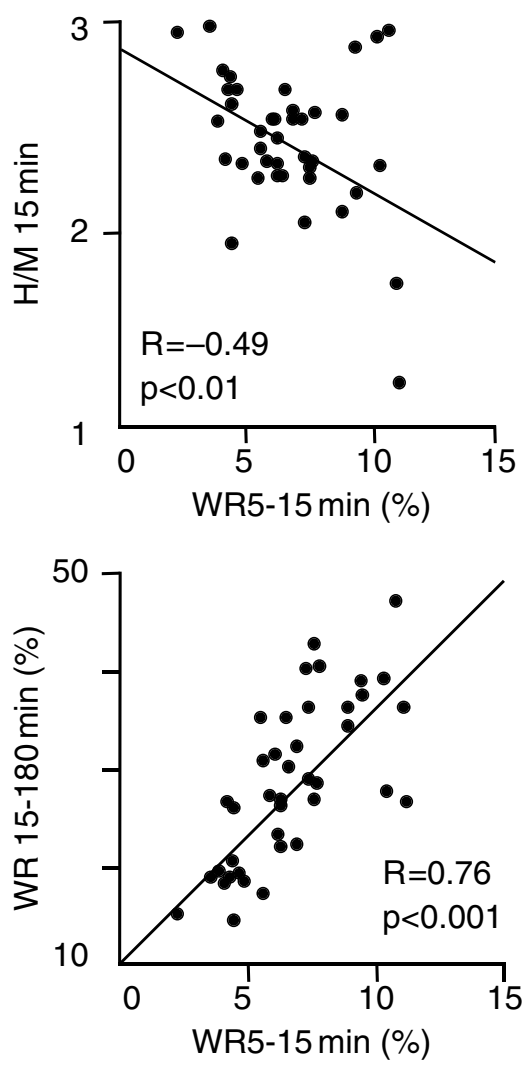
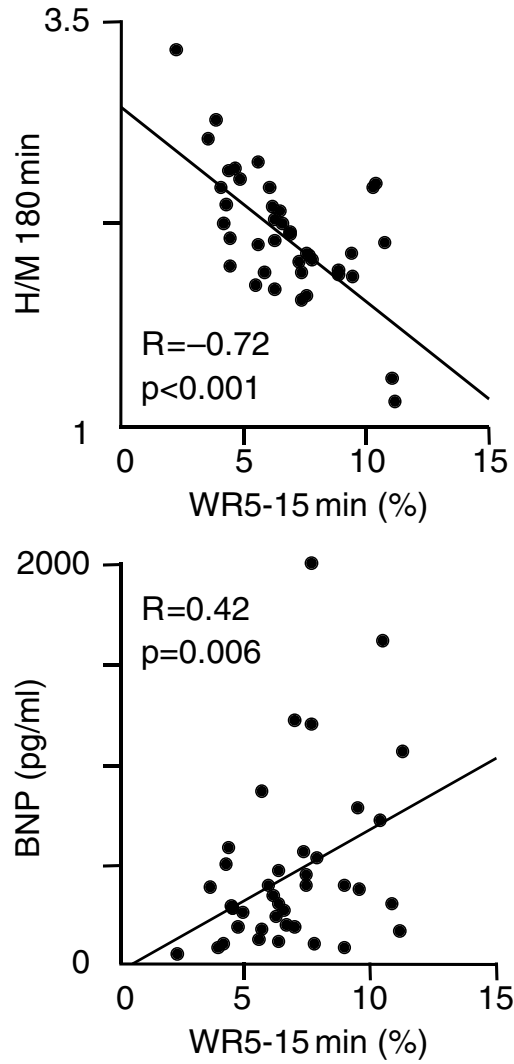

Fig 3. Correlations between the washout rate of iodine-123-metaiodobenzylguanidine from 5 to $15 \mathrm{~min}$ (WR5-15 $\mathrm{min}$ ) and general MIBG parameters and the plasma level of B-type natriuretic peptide (BNP). H/M, heart to mediastinum. prognostic factors of cardiac events.

\section{Identification of Subgroups of Patients With High Risk}

Stratification of patients on the basis of the combined cutoff values of WR5-15 min (6.4\%) and LVEF (30\%) is shown in Fig 5. The cardiac event rate in patients with WR5-15 min $\geq 6.4 \%$ and $\mathrm{LVEF} \leq 30 \%$ was $73 \%$. The cardiac event rate was $31 \%$ in patients with WR $5-15 \mathrm{~min} \geq 6.4 \%$ or LVEF $\leq 30 \%$. Notably, no cardiac events occurred in patients with
Table 3 Results of Multivariate Analysis for Predicting Cardiac Events

\begin{tabular}{lccc}
\hline \hline & $H R$ & $95 \%$ CI of HR & $p$ value \\
\hline WR5-15 min,$\geq 6.4 \%$ & 9.789 & $1.145-83.704$ & 0.0372 \\
LVEF, $\leq 30 \%$ & 5.401 & $1.397-20.883$ & 0.0145 \\
$B N P, \geq 260 \mathrm{pg} / \mathrm{ml}$ & 0.792 & $0.202-3.107$ & NS
\end{tabular}

Abbreviations see in Tables 1,2.
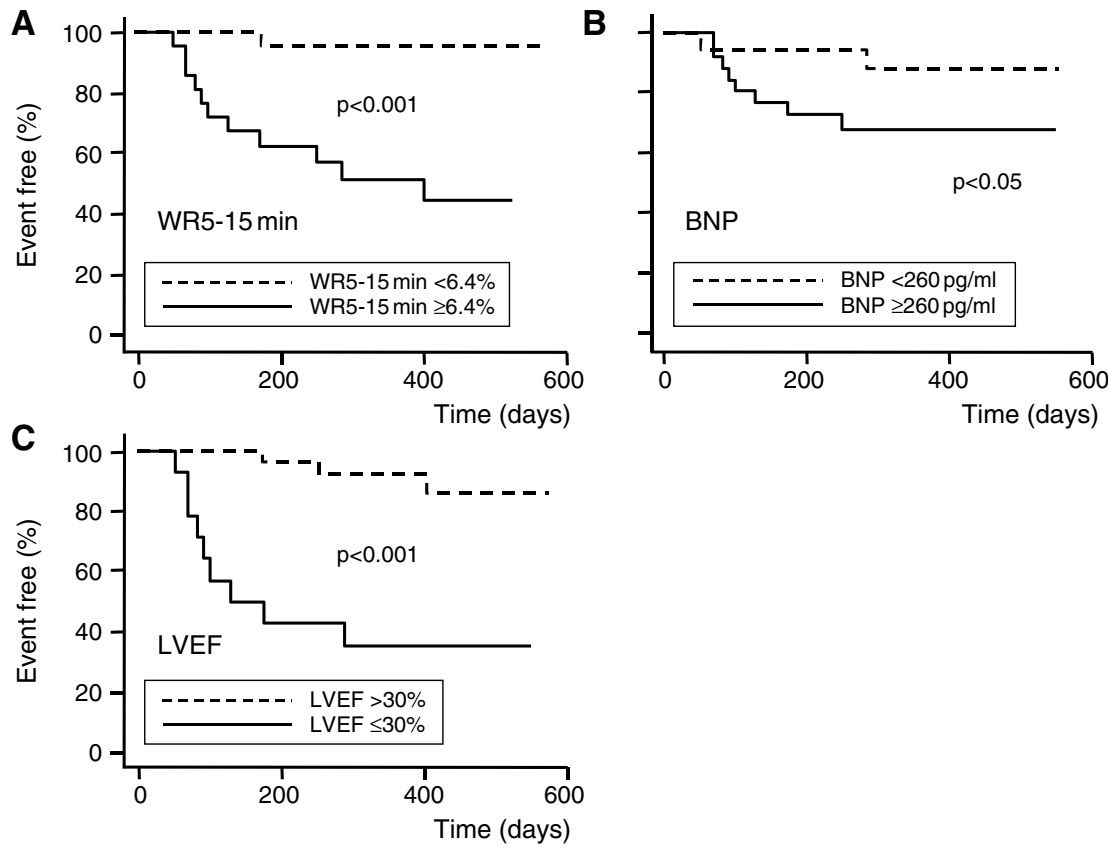

Fig 4. (A) Washout rate of iodine-123metaiodobenzylguanidine (123 I-MIBG) from 5 to $15 \mathrm{~min}$ (WR5-15 min) and all cardiac events in patients with heart failure. (B) Plasma levels of B-type natriuretic peptide (BNP) and cardiac events in patients with heart failure. (C) Left ventricular ejection fraction (LVEF) and cardiac events in patients with heart failure. Survival curves were created by Kaplan-Meier method. 


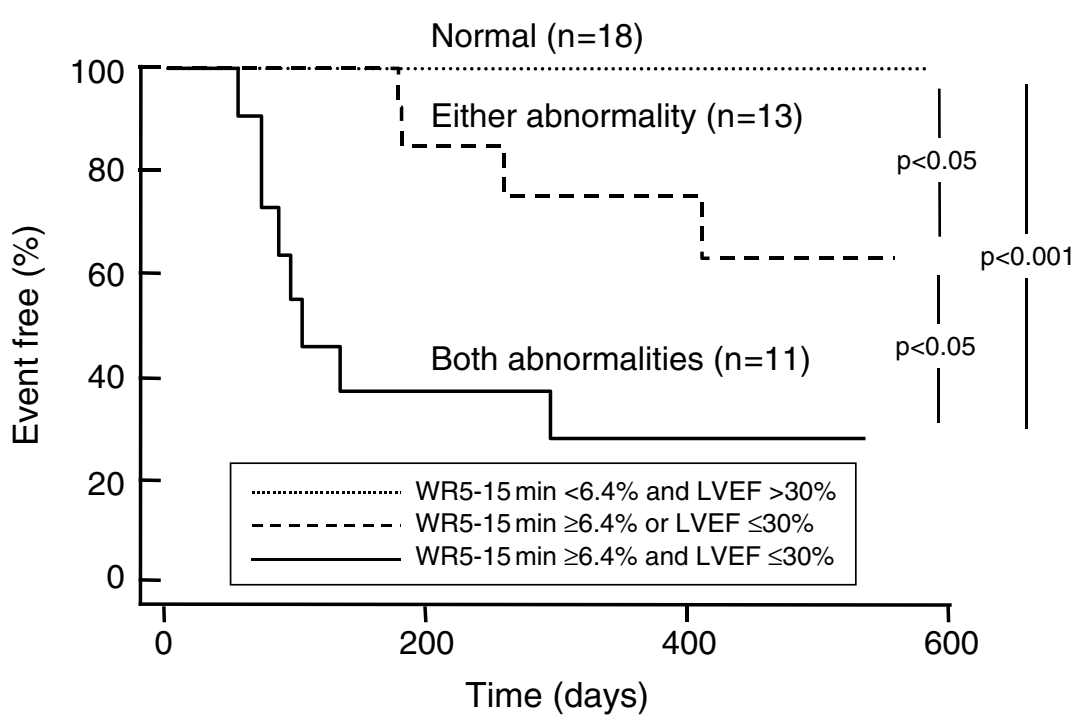

Fig 5. Kaplan-Meier analysis of cardiac-eventfree survival in patients with heart failure stratified into normal, either abnormality, or both abnormalities groups, based on the combination of the washout rate of iodine-123-metaiodobenzylguanidine (123 I-MIBG) from 5 to $15 \mathrm{~min}$ (WR5-15 min) and left ventricular ejection fraction (LVEF). Normal group: WR5-15 min $<6.4 \%$ and LVEF $>30 \%$; either abnormality group: WR5- $15 \mathrm{~min} \geq 6.4 \%$ or $\mathrm{LVEF} \leq 30 \%$; and both abnormalities group: WR5-15 $\mathrm{min} \geq 6.4 \%$ and $\mathrm{LVEF} \leq 30 \%$.
WR5-15 $\min <6.4 \%$ and LVEF $>30 \%$.

\section{Discussion}

This study has 2 interesting findings. (1) Our novel index, WR5-15 min, was augmented by NYHA functional class. WR5-15 min significantly correlated with conventional ${ }^{123}$ I-MIBG parameters such as WR15-180 min and H/M ratio. (2) Multivariate analysis showed WR5-15 min was an independent predictor for subsequent cardiac events. WR515 min provided additional prognostic information to that given by LVEF in patients with HF. These findings support the clinical effectiveness of a short ${ }^{123}$ I-MIBG imaging protocol, which also reduces the burden on the patient with HF.

Assessing cardiac autonomic status is clinically important in the management of patients with $\mathrm{HF}$, because sympathetic nervous activation causes excessive ventricular afterload, downregulation of $\beta$-adrenergic receptors, tachycardiac interference with ventricular filling, HF progression, and increased mortality. ${ }^{23}$ We and others have demonstrated that several parameters of ${ }^{123}$ I-MIBG imaging, such as H/M ratio and conventional washout rate, provide promising information for the assessment of patients with HF,9,9,17,18,20 but the long protocol for such imaging may limit this efficacious examination in some clinical settings. We recently demonstrated that washout of ${ }^{123}$ I-MIBG occurred 4 min after tracer injection and was already augmented in the early stage of HF, even in patients in NYHA functional classes I and II. ${ }^{17}$ In the present study, we evaluated ${ }^{123}{ }^{2}$-MIBG washout in the very early phase using anterior planar imaging, which is a simple and widely available method. Rapid ${ }^{123}$ IMIBG washout from 5 to $15 \mathrm{~min}$ was observed in patients with cardiac events; in fact, the WR5-15 min advanced with NYHA functional class. As shown Fig 3, WR5-15 min inversely correlated with H/M $180 \mathrm{~min}$ and positively correlated with WR15-180 min. WR5-15 min also positively correlated with the plasma level of BNP. These findings suggest that WR5-15 min reflects the functional severity of HF. Rapid ${ }^{123}$ I-MIBG washout in the very early phase may be a feature of failing myocardium, although the mechanism is still not apparent from our results. However, neither the number of patients nor the cardiac hard events were large enough and the follow-up period was not long enough, compared with previous studies, $5,9,17,18,20$ so the prognostic relevance of WR5-15 min remains conjectural.

It is now widely recognized that many patients with signs and symptoms of HF have preserved LVEF 24,25 The overall mortality and rate of HF hospitalization in patients with systolic dysfunction or preserved systolic function are very similar.5 On the other hand, LVEF is an important predictor of fatal and nonfatal cardiovascular outcomes, including HF hospitalization, sudden death and HF death, in those with a severe reduction of LV systolic function! ${ }^{16}$ In agreement with those results, the rate of cardiac events was markedly higher in patients with severe systolic dysfunction as shown in Fig 4.

We and others have documented the rationale for combined evaluation of cardiac nervous assessment and biomarkers for risk stratification of patients with HF $18,20 \mathrm{We}$ can detect high-risk patients with HF using a sole adverse factor, whereas the predictive power to detect low-risk patients is insufficient. In the present study cardiac events occurred in some patients stratified into the low-risk group (Fig 4). Combined assessment may contribute to a more precise stratification of low-risk patients compared with a single assessment. Interestingly, no cardiac events occurred in patients with both preserved WR5-15 min and LVEF.

\section{Study Limitations}

Several potential limitations should be considered with respect to these findings. First, the patients were treated with medications that may have affected cardiac MIBG uptake, such as $\beta$-adrenergics, angiotensin-converting enzyme inhibitors, angiotensin-II receptor blockers, and spironolactone $22,26,27$ Second, we also acknowledge the fact that other MIBG parameters (WR15-180 min and H/M $180 \mathrm{~min}$ ), and BNP did not predict cardiac events independently in the present study. Presumably, our results do not apply to severe HF because $43 \%$ of the patients were in NYHA class II. Additionally, the number of patients and the number of adverse cardiac accidents were not large enough. Only 2 patients died from progressive HF and 1 patient died suddenly. In this respect, a large-scale completely prospective study is needed to further assess the prognostic value of a short ${ }^{123}$ I-MIBG imaging protocol. Third, the washout curve of cardiac ${ }^{123}$ I-MIBG was steeper in the very early phase compared with the late phase ${ }^{17}$ A slight time lag in 
image acquisition may affect the calculation of 123 I-MIBG washout in the very early phase, because the SD of WR515 min was relatively large compared with that for WR15$180 \mathrm{~min}$.

\section{Conclusions}

WR5-15 min obtained with anterior planar imaging is useful to evaluate the severity and clinical outcome of HF. We may be able to shorten the ${ }^{123}$ I-MIBG imaging protocol and thus lighten the patient's burden with this novel process.

\section{References}

1. O'Connor CM. The new heart failure guidelines: Strategies for implementation. Am Heart J 2007; 153: 2-5.

2. McMurray J, Cohen-Solal A, Dietz R, Eichhorn E, Erhardt L, Hobbs FD, et al. Practical recommendations for the use of ACE inhibitors, beta-blockers, aldosterone antagonists and angiotensin receptor blockers in heart failure: Putting guidelines into practice. Eur J Heart Fail 2005; 7: 710-721.

3. Bristow MR, Saxon LA, Boehmer J, Krueger S, Kass DA, De Marco $\mathrm{T}$, et al. Cardiac-resynchronization therapy with or without an implantable defibrillator in advanced chronic heart failure. $N$ Engl $J$ Med 2004; 350: 2140-2150.

4. Wong M, Staszewsky L, Latini R, Barlera S, Glazer R, Aknay N, et al. Severity of left ventricular remodeling defines outcomes and response to therapy in heart failure: Valsartan heart failure trial (ValHeFT) echocardiographic data. J Am Coll Cardiol 2004; 43: $2022-$ 2027.

5. Nakata T, Miyamoto K, Doi A, Sasao H, Wakabayashi T, Kobayashi $\mathrm{H}$, et al. Cardiac death prediction and impaired cardiac sympathetic innervation assessed by MIBG in patients with failing and nonfailing hearts. J Nucl Cardiol 1998; 5: 579-590.

6. Sakai H, Tsutamoto T, Tsutsui T, Tanaka T, Ishikawa C, Horie M. Serum level of uric acid, partly secreted from the failing heart, is a prognostic marker in patients with congestive heart failure. Circ $J$ 2006; 70: $1006-1011$

7. Ishikawa C, Tsutamoto T, Fujii M, Sakai H, Tanaka T, Horie M. Prediction of mortality by high-sensitivity $\mathrm{C}$-reactive protein and brain natriuretic peptide in patients with dilated cardiomyopathy. Circ J 2006; 70: $857-863$.

8. Niizeki T, Takeishi Y, Arimoto T, Nozaki N, Hirono O, Watanabe T, et al. Persistently increased serum concentration of heart-type fatty acid-binding protein predicts adverse clinical outcomes in patients with chronic heart failure. Circ J 2008; 72: 109-114.

9. Wakabayashi T, Nakata T, Hashimoto A, Yuda S, Tsuchihashi K, Travin MI, et al. Assessment of underlying etiology and cardiac sympathetic innervation to identify patients at high risk of cardiac death. J Nucl Med 2001; 42: 1757-1767.

10. McDonagh TA, Robb SD, Murdoch DR, Morton JJ, Ford I, Morrison $\mathrm{CE}$, et al. Biochemical detection of left-ventricular systolic dysfunction. Lancet 1998; 351: 9-13.

11. Anand IS, Fisher LD, Chiang YT, Latini R, Masson S, Maggioni AP, et al. Changes in brain natriuretic peptide and norepinephrine over time and mortality and morbidity in the Valsartan Heart Failure Trial (Val-HeFT). Circulation 2003; 107: 1278-1283.
12. Perna ER, Macin SM, Canella JP, Augier N, Stival JL, Cialzeta JR, et al. Ongoing myocardial injury in stable severe heart failure: Value of cardiac troponin T monitoring for high-risk patient identification. Circulation 2004; 110: 2376-2382.

13. Troughton RW, Frampton CM, Yandle TG, Espiner EA, Nicholls MG, Richards AM. Treatment of heart failure guided by plasma aminoterminal brain natriuretic peptide (N-BNP) concentrations. Lancet 2000; 355: 1126-1130.

14. Kuwabara Y, Sato Y, Miyamoto T, Taniguchi R, Matsuoka T, Isoda $\mathrm{K}$, et al. Persistently increased serum concentrations of cardiac troponin in patients with acutely decompensated heart failure are predictive of adverse outcomes. Circ J 2007; 71: 1047-1051.

15. Tsutamoto T, Sakai H, Nishiyama K, Tanaka T, Fujii M, Yamamoto $\mathrm{T}$, et al. Direct comparison of transcardiac increase in brain natriuretic peptide (BNP) and $\mathrm{N}$-terminal proBNP and prognosis in patients with chronic heart failure. Circ J 2007; 71: 1873-1878.

16. Solomon SD, Anavekar N, Skali H, McMurray JJ, Swedberg K, Yusuf S, et al. Influence of ejection fraction on cardiovascular outcomes in a broad spectrum of heart failure patients. Circulation 2005; 112: $3738-3744$.

17. Arimoto T, Takeishi Y, Fukui A, Tachibana H, Nozaki N, Hirono O, et al. Dynamic ${ }^{123}$ I-MIBG SPECT reflects sympathetic nervous integrity and predicts clinical outcome in patients with chronic heart failure. Ann Nucl Med 2004; 18: 145-150.

18. Arimoto T, Takeishi Y, Niizeki T, Nozaki N, Hirono O, Watanabe T, et al. Cardiac sympathetic denervation and ongoing myocardial damage for prognosis in early stages of heart failure. J Card Fail 2007; 13: $34-41$.

19. Ogita H, Shimonagata T, Fukunami M, Kumagai K, Yamada T, Asano Y, et al. Prognostic significance of cardiac (123)I metaiodobenzylguanidine imaging for mortality and morbidity in patients with chronic heart failure: A prospective study. Heart 2001; 86: 656-660.

20. Kyuma M, Nakata T, Hashimoto A, Nagao K, Sasao H, Takahashi T, et al. Incremental prognostic implications of brain natriuretic peptide, cardiac sympathetic nerve innervation, and noncardiac disorders in patients with heart failure. J Nucl Med 2004; 45: 155-163.

21. Arimoto T, Takeishi Y, Niizeki T, Koyama Y, Okuyama H, Nozaki $\mathrm{N}$, et al. Ongoing myocardial damage relates to cardiac sympathetic nervous disintegrity in patients with heart failure. Ann Nucl Med 2005; 19: $535-540$.

22. Takeishi Y, Atsumi H, Fujiwara S, Takahashi K, Tomoike H. ACE inhibition reduces cardiac iodine-123-MIBG release in heart failure. J Nucl Med 1997; 38: 1085-1089.

23. Schrier RW, Abraham WT. Hormones and hemodynamics in heart failure. N Engl J Med 1999; 341: 577-585.

24. Kitahara T, Takeishi Y, Arimoto T, Niizeki T, Koyama Y, Sasaki T, et al. Serum carboxy-terminal telopeptide of type I collagen (ICTP) predicts cardiac events in chronic heart failure patients with preserved left ventricular systolic function. Circ J 2007; 71: 929-935.

25. Chatterjee K, Massie B. Systolic and diastolic heart failure: Differences and similarities. J Card Fail 2007; 13: 569-576.

26. Kasama S, Toyama T, Hatori T, Sumino H, Kumakura H, Takayama $\mathrm{Y}$, et al. Evaluation of cardiac sympathetic nerve activity and left ventricular remodelling in patients with dilated cardiomyopathy on the treatment containing carvedilol. Eur Heart J 2007; 28: 989-995.

27. Kasama S, Toyama T, Sumino H, Matsumoto N, Sato Y, Kumakura $\mathrm{H}$, et al. Additive effects of spironolactone and candesartan on cardiac sympathetic nerve activity and left ventricular remodeling in patients with congestive heart failure. J Nucl Med 2007; 48: 1993-2000. 